\title{
Multichannel active control of random noise in a small reverberant room
}

\section{Laugesen, Søren; Elliott, Stephen J.}

Published in:

IEEE transactions on speech and audio processing

Link to article, DOI:

$10.1109 / 89.222881$

Publication date:

1993

Document Version

Publisher's PDF, also known as Version of record

Link back to DTU Orbit

Citation (APA):

Laugesen, S., \& Elliott, S. J. (1993). Multichannel active control of random noise in a small reverberant room. IEEE transactions on speech and audio processing, 1(2), 241-249. https://doi.org/10.1109/89.222881

\section{General rights}

Copyright and moral rights for the publications made accessible in the public portal are retained by the authors and/or other copyright owners and it is a condition of accessing publications that users recognise and abide by the legal requirements associated with these rights.

- Users may download and print one copy of any publication from the public portal for the purpose of private study or research.

- You may not further distribute the material or use it for any profit-making activity or commercial gain

- You may freely distribute the URL identifying the publication in the public portal

If you believe that this document breaches copyright please contact us providing details, and we will remove access to the work immediately and investigate your claim. 


\title{
Multichannel Active Control of Random Noise in a Small Reverberant Room
}

\author{
Søren Laugesen and Stephen J. Elliott, Member, IEEE
}

\begin{abstract}
In this study an algorithm for multichannel adaptive IIR filtering is presented and applied to the active control of broadband random noise in a small reverberant room. Assuming complete knowledge of the primary noise, the theoretically optimal reductions of acoustic energy are initially calculated by means of a frequency-domain modal model. These results are contrasted with results of a causality constrained theoretical timedomain optimization, which are then compared with experimental results, the latter two results showing good agreement. The experimental performance of adaptive multichannel FIR and IIR filters are then compared for a 4-secondary source, 8-error microphone active control system, indicating that for the present application FIR filters are sufficient when the primary noise source is a loudspeaker. Some experiments are then presented with the primary noise field generated by a panel excited by a loudspeaker in an adjoining room. These results show that far better performances are provided by IIR than FIR filters when the primary source has a lightly damped dynamic behavior which the active controller must model.
\end{abstract}

\section{INTRODUCTION}

$\mathbf{I}$ $\mathrm{N}$ single channel active systems for the control of broadband acoustic plane waves propagating in a duct, it is well known that IIR filters are capable of matching the complicated response of the desired controller with fewer filter coefficients than FIR filters [1], [2]. Despite the potential stability problems associated with IIR filters [3], successful practical applications have been reported [1], [2], [4].

In rooms and enclosures, where the sound field is more complicated, multichannel active methods must generally be used when global control of the sound field is the objective. Practical versions of such systems so far have demonstrated only substantial and reliable reductions in the case of repetitive noise with few harmonics. The best known applications are the control of "boom" noise in cars [5] and the control of propeller-induced noise in flight cabin interiors [6].

There is, however, also a need to control broadband nonrepetitive (random) noise at low frequencies in rooms. Most structures have a small transmission loss at low frequencies, and there are several low-frequency noise sources in our environment with random waveforms, such as passing traffic and trains, noisy neighbors etc. There are many issues to be addressed before it can be decided whether such problems are

Manuscript received August 27, 1991; revised March 31, 1992. The associate editor coordinating the review of this paper and approving it for publication was Dr. Sharad Singhal.

S. Laugesen is with The Acoustics Laboratory, Technical University of Denmark, Building 352, DK-2800 Lyngby, Denmark.

S. J. Elliott is with the Institute of Sound and Vibration Research, University of Southampton, GB-SO9 5NH Southampton, England.

IEEE Log Number 9206395 amenable to active control. However, active noise control is known to work best at low frequencies, and with the rapid developments in DSP devices it seems timely to begin such an enquiry.

The objective of this investigation is to demonstrate that an adaptive algorithm for multichannel IIR filters can be used for the active control of a broadband non-repetitive noise in a reverberant room. Practical implementation of true multichannel adaptive IIR filtering in acoustic applications had, to the authors' knowledge, not been reported prior to the first report of the current work [15], although an active control system with two independent IIR filters for control of higher order modes in a duct had been described by Eriksson et al. [7]. Apart from some experimental results on the control of road noise in cars using adaptive FIR filters presented by McDonald et al. [8], active control of broadband noise in enclosures had previously been treated only theoretically [9]-[11].

Based on the experience gained from the duct problem mentioned above, it would be expected that a multichannel active control system for broadband noise featuring IIR control filters would stand a better chance of globally controlling the sound field than a system featuring the FIR filters that have been used previously in multichannel systems for repetitive noise [12].

For the practical demonstration a small rectangular reverberant room was chosen. The room measured $2.53 \mathrm{~m} \times 2.51$ $\mathrm{m} \times 2.08 \mathrm{~m}$ and had a reverberation time of about $1 \mathrm{~s}$ in the low-frequency range. The room was actually the receiver room in a transmission loss suite, and hence it was connected to another small reverberant room by a square window (side length $0.685 \mathrm{~m}$ ) in the centre of one of the $2.53 \mathrm{~m} \times 2.51 \mathrm{~m}$ walls. All simulations presented in this study pertain to this particular room, although the theory of course applies to any room or enclosure.

The remaining parts of this paper are set out as follows. First, the theoretical maximum reductions of the sum of the squares of the pressures at the error sensors after active control are calculated from a frequency-domain modal model, and compared with the resulting reduction in acoustic potential energy in the room. Since the optimal complex source strengths obtained from this minimization correspond to noncausal time functions, these results are compared with the results of an optimization performed under the constraint of a causal controller. Secondly, the multichannel adaptive IIR filter algorithm is derived, and finally the experimental results, obtained using the aforementioned algorithm, are presented and discussed. 


\section{Theoretical Predictions}

The performance of a practical active noise control system will depend on both the fundamental physical constraints imposed by the acoustic arrangement, and on the behavior of the control algorithm used. In order to establish a benchmark for the reductions in sound level obtained experimentally, theoretical predictions of the acoustic limitations of active control will be considered in this section.

The foundation of the theoretical predictions is a frequencydomain modal model of the sound field in the room, as described by Nelson et al. [13]. The complex sound pressure at a point in the room, due to a harmonic source with a certain velocity distribution, is expressed as the sum of a number of modal resonance terms. The sources are divided into a "primary" source, generating the undesired noise, and "secondary" sources, which are controlled such that the primary and secondary fields interfere destructively. Each source is modeled as a square piston mounted in one of the walls in the room and driven at an angular frequency $\omega$. By means of this model the pressure at the error microphones can be expressed as

$$
\boldsymbol{p}_{E}(\omega)=\boldsymbol{p}_{P}(\omega)+\boldsymbol{Z}(\omega) \cdot \boldsymbol{q}(\omega)
$$

where $p_{E}(\omega)$ is the vector of the total complex sound pressures at the $L$ error sensors, $p_{P}(\omega)$ is the vector of sound pressures at the error sensors due to the primary source alone, $q(\omega)$ is the vector of $M$ complex secondary source strengths, and the $Z(\omega)$ matrix consists of acoustic transfer impedances calculated from the model outlined above.

\section{A. Unconstrained Optimization}

When carrying out this unconstrained optimization, the system is considered to be in the steady state excited at a single frequency $(\omega)$. The problem will then be to choose the complex secondary source strengths $q(\omega)$ that minimize the sum of the squares of the pressures at the error sensors, that is, the quantity [14]:

$$
\begin{aligned}
J_{p}(\omega) & =\boldsymbol{p}_{E}(\omega)^{H} \boldsymbol{p}_{E}(\omega) \\
& =\left[\boldsymbol{p}_{P}(\omega)+\boldsymbol{Z}(\omega) \cdot \boldsymbol{q}(\omega)\right]^{H}\left[\boldsymbol{p}_{P}(\omega)+\boldsymbol{Z}(\omega) \cdot \boldsymbol{q}(\omega)\right]
\end{aligned}
$$

where ${ }^{H}$ denotes the Hermitian complex transpose. This minimization problem is clearly quadratic, and has the unique solution [14]:

$$
\boldsymbol{q}_{o}(\omega)=-\left[\boldsymbol{Z}(\omega)^{H} \boldsymbol{Z}(\omega)\right]^{-1} \boldsymbol{Z}(\omega)^{H} \boldsymbol{p}_{P}(\omega) .
$$

According to [14] the resulting minimum value of $J_{p}$ is

$$
\begin{aligned}
J_{p o}(\omega)= & {\left[\boldsymbol{p}_{P}(\omega)^{H} \boldsymbol{p}_{P}(\omega)\right.} \\
& \left.-\boldsymbol{p}_{P}(\omega)^{H} \boldsymbol{Z}(\omega)\left[\boldsymbol{Z}(\omega)^{H} \boldsymbol{Z}(\omega)\right]^{-1} \boldsymbol{Z}(\omega)^{H} \boldsymbol{p}_{P}(\omega)\right]
\end{aligned}
$$

which should be compared to the uncontrolled value:

$$
J_{p p}(\omega)=\boldsymbol{p}_{P}(\omega)^{H} \boldsymbol{p}_{P}(\omega) .
$$

However, in terms of the physical effect of control the sum of squared pressures at the error microphones does not constitute the entire story, since a reduction in $J_{p}$, based on an unfortunate distribution of error sensors and/or secondary sources, might well be accompanied by an increase in the acoustic potential energy in the enclosure. This has been demonstrated by Bullmore et al. [14] and by Laugesen and Elliott [15]. The real test of the optimization is to calculate the residual acoustic potential energy resulting from the introduction of the secondary source strengths calculated in (3). In [13] it was demonstrated how the total time averaged acoustic potential energy in the enclosure,

$$
E_{p}(\omega)=\left(1 / 4 \rho c^{2}\right) \cdot \int_{V}|p(x, \omega)|^{2} d V
$$

could be calculated from the modal model. In (6), $\rho$ is the density of air, $c$ the speed of sound, and $V$ the volume of the room. In a fashion similar to that above, the theoretical minimization of $E_{p}$ can be carried out [13]. Thus it can be investigated how close minimizing the sum of the squared pressures at the microphones $\left(J_{p}\right)$ comes to the true objective of minimizing the total acoustic potential energy $\left(E_{p}\right)$.

The number of error sensors and secondary sources used for the control system will in practice be limited by the capability of the signal processing hardware. Bearing in mind the hardware which was available for the present experiments, and acknowledging that a sensible proportion of secondary sources to error sensors is around $1: 2$ [14], a system with $M=4$ secondary sources and $L=8$ error sensors was chosen for the simulations. Following the investigations of Bullmore et al. [14], the error sensors were positioned one in each corner of the rectangular room. The primary source was positioned in the window opening, slightly off the centre of one of the 2.51 $\mathrm{m} \times 2.53 \mathrm{~m}$ walls. After considering 12 different distributions of the secondary sources in the room, the secondary sources were positioned on the floor, one near each corner a distance of $30-50 \mathrm{~cm}$ away from the walls. This arrangement was easy to use in practice, and gave reductions which were nearly as good as any other distribution tried.

The model and the optimization routines discussed above was implemented in a simulation program running on a PC and are detailed in [15]. The results of the optimizations for sinusoidal excitation over a range of frequencies are presented in Figs. 1 and 2. Note that there is a fairly good agreement between the reduction at the microphones $J_{p}$ and the reduction in the energy in the room $E_{p}$ below $120 \mathrm{~Hz}$, whereas the reduction at the microphones is not followed by a similar reduction in energy for higher frequencies. Furthermore, it is seen from Fig. 2 that for frequencies under $150 \mathrm{~Hz}$, similar reductions in $E_{p}$ are obtained whether the actual energy in the room or the sum of the squared pressures at the error sensors is minimized, whereas for higher frequencies better reductions result from minimizing the energy. This indicates that for frequencies below $150 \mathrm{~Hz}, 8$ microphones positioned in the corners of the room provide a good estimate of the energy in the room in this case. The results shown are typical for a "well-conditioned" distribution of secondary sources, in the sense that no substantial increase in the energy occurs in any frequency range, and in the sense that the secondary source strengths are of reasonable magnitudes compared to the strength of the primary source, as can be seen in Fig. 3 . In this figure, $0 \mathrm{~dB}$ corresponds to the level of the strength of 


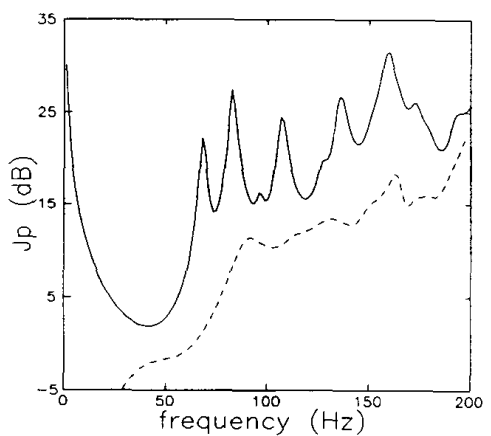

Fig. 1. The predicted sum of the squares of the sound pressures at the 8 error sensor positions in the room $\left(J_{p}\right)$ before and --- after minimization at each frequency using the computer model, with 4 secondary sources positioned on the floor.

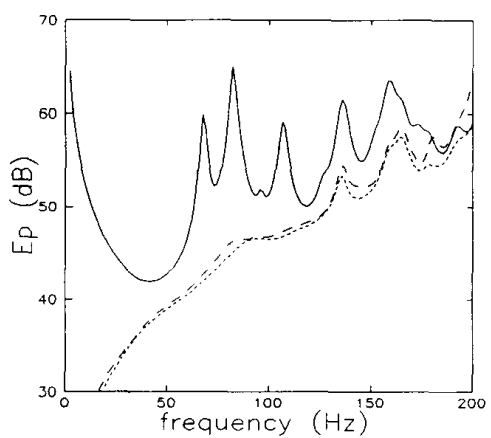

Fig. 2. The predicted total acoustic potential energy $\left(E_{p}\right)$ in the room before and - - - after control with 4 secondary sources acting so as to minimize the sum of the squares of the sound pressures at the 8 error sensors, and $\cdots \cdots$ after minimization of the energy $\left(E_{p}\right)$ with the same secondary source configuration.

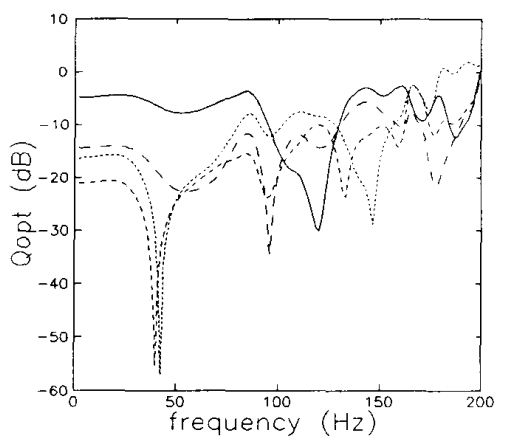

Fig. 3. The modulus of the source strengths of the 4 secondary sources relative to that of the primary source, corresponding to Fig. 1 .

the primary source, and it is apparent that all the secondary source strengths are below that level at nearly all frequencies.

\section{B. Causality Constrained Optimization}

It is shown in [14]-[16] that if the optimal complex secondary source strengths that result from the unconstrained frequency-domain optimization over the whole frequency range, were derived from the primary source via filters, then these filters would have highly noncausal impulse responses. Hence, it is important also to carry out an optimization under the constraint of causality. This has previously been done by Nelson et al. [10] and Joplin and Nelson [11] for the continuous-time case, and by Hough [16] for the discrete-time case. The theory used here can be found in Elliott et al. [12], and was implemented in a suite of programs developed by Sutton [17], parts of which were used in this study.

In order to facilitate this optimization it is necessary to assume that the signal driving the primary source is available as an electrical input $x(n)$, called the reference signal. The starting point of the time-domain optimization is then the discrete time-domain equivalent of (1), separated into $L$ scalar equations, one for each sample error signal $e_{l}(n)$ :

$$
e_{1}(n)=d_{1}(n)+\sum_{m=1}^{M} \sum_{j=0}^{J-1} c_{l m j} \sum_{i=0}^{I-1} a_{m}(i) x(n-i-j) .
$$

The first term in the right side of (7) is the contribution of the primary source to the $l$ th error microphone. The second term is the contribution from the secondary sources, where $c_{l m j}$ are the coefficients of the Jth-order FIR filters representing the impulse response from the $m$ th secondary source to the $l$ th error microphone and $a_{m}(i)$ are the coefficients of the Ithorder causal FIR filters, which are assumed to constitute the controllers in this case. The expectation of the sum of the squares of the error signals,

$$
J=E\left\{\sum_{l=1}^{L} e_{1}(n)^{2}\right\}
$$

can now be minimized with respect to all the filter coefficients $a_{m}(i)$. This is another quadratic minimization problem [12], [16], again with a unique optimal solution denoted $a_{o, m}(i)$.

An important point is that the optimal filters depend on the autocorrelation function of the reference signal; hence it should be emphasized that after introducing the constraint of causality in the optimization, it has no meaning to speak of the attenuation at a single frequency without specifying the waveform of the reference signal.

For the configuration of loudspeakers and microphones used above and a sampling frequency of $f_{s}=400 \mathrm{~Hz}$, the primary path and error path frequency responses calculated from the modal model were Fourier transformed into impulse responses and used to generate $d_{1}(n)$ and $c_{l m j}$. In the case of a white noise primary signal $x(n)$, the sum of the squared error signals (see (8)) was minimized by adjusting the 128 coefficient of each of the four causal FIR control filters. The result is shown in Fig. 4 where each optimal filter occupies a 128 sample block on the horizontal axis. It is clearly sufficient to use only 128 coefficients for each filter, in order for them to decay nearly to zero in this white noise excitation case, indicating that further increases in filter length would not significantly decrease the cost function.

The sum of the power spectral densities at the 8 microphones (denoted PSD8) was calculated, since that is what will actually be measured in the experiments. This quantity is plotted in Fig. 5. It should be emphasized that in contrast to the 


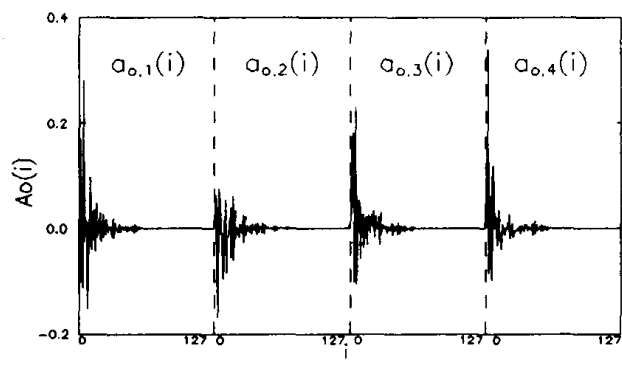

Fig. 4. The impulse responses of the 4 optimal 128-point causal FIR control filters, found by minimization of the expectation of the sum of the squared error signals with the computer model in the case of a white noise reference signal.

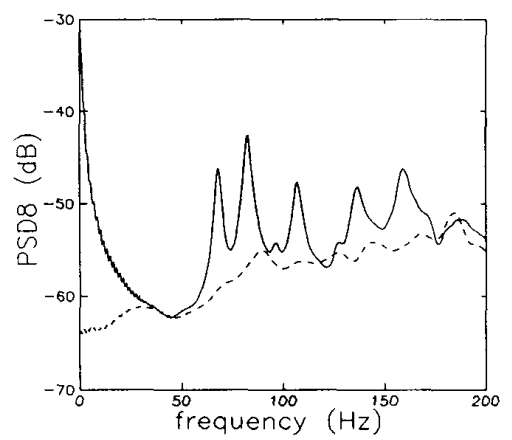

Fig. 5. The predicted sum of the power spectral densities of the 8 error signals (PSD8) before and - - - after control with causality constrained FIR filters (Fig. 4) and a white noise reference signal.

reductions predicted from the frequency-domain optimization above, the reductions at all frequencies shown in Fig. 5 are obtained simultaneously, with a single broadband random excitation signal. Furthermore, the optimal causal filters were transformed back to the frequency domain, and then used in the modal model to also calculate the residual energy $E_{p}$, with the result shown in Fig. 6 . From both Figs. 5 and 6 it can be seen that the constraint of causality somewhat degrades the performance of the active control system compared with that of the unconstrained minimization, cf. Figs. 1 and 2. However, there is still a considerable reduction of the resonance peaks, and only slight increases in two of the gaps between resonances. It is encouraging to notice that the reductions at the microphones (PSD8) are very close to the reductions in the total acoustic potential energy in the room $\left(E_{p}\right)$.

In order to demonstrate the significance of the "predictability" of the reference signal, and to obtain a result from the simulations which is comparable with the experimental results to be presented below, the causality constrained optimization was repeated with a number of different reference signals. These reference signals were taken to be white noise filtered through a specified transfer function, so that the power spectral density of the noise was simply the squared modulus of the transfer function in question. By Fourier transforming the power spectral densities, the autocorrelation function of the reference signal was found and used in the constrained optimization described above. The result calculated in this

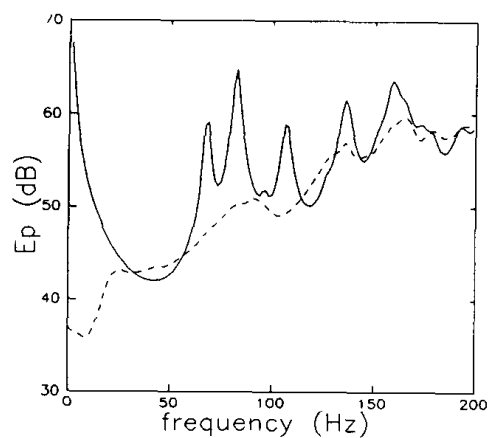

Fig. 6. The predicted total acoustic potential energy $\left(E_{p}\right)$ in the room before and - - - after control with causality constrained FIR filters and a white noise reference signal as in Fig. 5 .

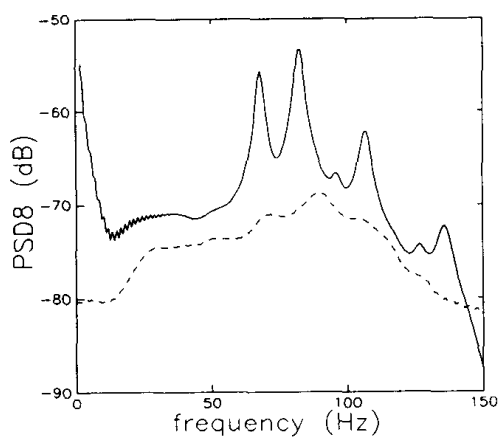

Fig. 7. The predicted sum of the power spectral densities of the 8 error signals (PSD8) calculated from the computer simulation before and - - after control with causality constrained FIR filters and a "colored" reference signal, with a spectrum similar to that used experimentally.

way that most resembled the results obtained in the initial experiments (presented in Section IV-B), in terms of the uncontrolled value of PSD8, is shown in Fig. 7. Note that the frequency scale has been somewhat expanded in this case for comparison with the experimental results below. It can be seen that the reductions for this "colored" noise are somewhat better in the $20-100-\mathrm{Hz}$ range than those obtained in the white noise case, because the filters can expend more "effort" in the frequency range that contributes more to the mean square error.

It is clear that the potential reductions which could be obtained in the sound field would increase if it was possible to introduce additional delay into the primary paths. This could be achieved, for example, by obtaining the reference signal from further upstream in an air conditioning duct, if this was the primary source of the noise. The performance of the active control system could then be improved, up to the limits imposed by the unconstrained optimization (Fig. 1). If, however, an additional delay is introduced in the secondary paths $\left(c_{1 m}\right)$, the performance will be degraded, as described by Sutton $e t$ al. [17]. Such an additional delay in the secondary paths would be introduced, for example, if detection sensors needed to be used to indirectly measure the output of the primary source, in which case the additional anti-aliasing filters and data converters would introduce delays in the control chain. 


\section{ADAPTIVE CONTROLLER}

In this section we develop an algorithm for adapting the coefficients of an array of IIR control filters in a multichannel active control system [18]. The algorithm uses an approximate steepest descent (stochastic gradient) method to minimize a cost function given by the instantaneous sum of the squared outputs from the $L$ error sensors

$$
J=\sum_{l=1}^{L} e_{1}^{2}(n)
$$

where each error signal, as in (7), is the superposition of contributions from the primary source $d_{1}(n)$ and each of the outputs from the $M$ secondary sources $y_{m}(n)$, so that

$$
e_{1}(n)=d_{1}(n)+\sum_{m=1}^{M} \sum_{j=0}^{J-1} c_{l m j} y_{m}(n-j)
$$

where $c_{l m j}$ are the coefficients of the $J$ th-order FIR filter representing the impulse response from the $m$ th secondary source to the $l$ th error sensor, as in (7), (although IIR filters could also be used to model this response).

In this general case the output of the $m$ th secondary source is made up of recursively filtered versions of $P$ reference signals, which may for example represent the outputs of $P$ primary sources, so that

$$
y_{m}(n)=\sum_{p=1}^{P} y_{p m}(n)
$$

where

$$
\begin{aligned}
y_{p m}(n)= & \sum_{i=0}^{I-1} a_{p m i}(n-1) x_{p}(n-i) \\
& +\sum_{k=1}^{K} b_{p m k}(n-1) y_{p m}(n-k)
\end{aligned}
$$

in which $x_{p}(n)$ is the $p$ th reference signal, $y_{p m}(n)$ is the filtered version of this reference signal used to drive the $m$ th secondary source, $a_{p m i}(n)$ is the direct coefficients of this filter, and $b_{p m k}(n)$ is the recursive coefficients of this filter, all at the $n$th sample time.

In deriving practical approximations to the required gradients of the cost function with respect to each of the filter coefficients, a number of rather sweeping assumptions must be made. To begin with, we assume that the control filter coefficients are time invariant so that the derivative of the cost function, with respect to a direct coefficient, can be written:

$$
\begin{aligned}
\frac{\partial J}{\partial a_{p m i}} & =2 \sum_{l=1}^{L} e_{1}(n) \frac{\partial e_{1}(n)}{\partial a_{p m i}} \\
& =2 \sum_{l=1}^{L} e_{1}(n) \sum_{j=0}^{J-1} c_{l m j} \frac{\partial y_{p m}(n-j)}{\partial a_{p m i}}
\end{aligned}
$$

which follows from (9)-(11). This assumption is valid as long as the filter coefficients vary slowly compared to the impulse response of the acoustical system, which is always the case in practice. Using (12), we can now write

$$
\frac{\partial y_{p m}(n)}{\partial a_{p m i}} \approx x_{p}(n-i)+\sum_{k=1}^{K} b_{p m k} \frac{\partial y_{p m}(n-k)}{\partial a_{p m i}} \text {. }
$$

The approximation in (14) arises because we ignore the acoustic feedback path which may, in the most general case, exist between the outputs of the secondary sources and the reference signals. Such feedback would make the reference signals themselves a function of the control filter coefficients. We now adapt the approach of Feintuch [19] in approximating the recursive form of (14) by the first term only, so that the gradient estimate from (13) can be written as

$$
\frac{\widehat{\partial J}}{\partial a_{p m i}}=2 \sum_{l=1}^{L} e_{1}(n) r_{p l m}(n-i)
$$

where the filtered reference signals, as introduced for the filtered-x LMS algorithm described, for example, by Widrow and Stearns [20], is defined to be

$$
r_{p l m}(n)=\sum_{j=0}^{J-1} c_{l m j} x_{p}(n-j)
$$

In a similar way, the derivative of the cost function with respect to the recursive filter coefficients can be written

$$
\frac{\partial J}{\partial b_{p m k}}=2 \sum_{l=1}^{L} e_{1}(n) \sum_{j=0}^{J-1} c_{l m j} \frac{\partial y_{p m}(n-j)}{\partial b_{p m k}}
$$

in which case, again from (12),

$$
\frac{\partial y_{p m}(n)}{\partial b_{p m k}} \approx y_{p m}(n-k)+\sum_{\kappa=1}^{K} b_{p m \kappa} \frac{\partial y_{p m}(n-\kappa)}{\partial b_{p m k}} \text {. }
$$

If we again approximate the recursive form of this derivative by the first term $y_{p m}(n-k)$, the gradient estimate from (17) can be written as

$$
\frac{\widehat{\partial J}}{\partial b_{p m k}}=2 \sum_{l=1}^{L} e_{1}(n) s_{p l m}(n-k) .
$$

In this case the signal $s_{p l m}$ is the filtered output signal, defined by

$$
s_{p l m}(n)=\sum_{j=0}^{J-1} c_{l m j} y_{p m}(n-j) .
$$

The gradient estimates of (15) and (19) are now used to update the control filter coefficients every sample using the method of steepest descent, so that

$$
\begin{aligned}
a_{p m i}(n+1)= & \gamma_{1} \cdot a_{p m i}(n) \\
& -\alpha_{1} \sum_{l=1}^{L} e_{1}(n) r_{p l m}(n-i) \\
b_{p m k}(n+1)= & \gamma_{2} \cdot b_{p m k}(n) \\
& -\alpha_{2} \sum_{l=1}^{L} e_{1}(n) s_{p l m}(n-k)
\end{aligned}
$$


where $\alpha_{1}$ and $\alpha_{2}$ are the convergence coefficients for the two sets of update equations. The quantities $\gamma_{1}$ and $\gamma_{2}$ are numbers slightly less than unity that cause the coefficients of the filter to "leak" away if the update term is small [20]. Their effect is similar to minimizing a cost function that includes control effort as well as total error [12]. It has been found that the inclusion of a leak has a very important stabilizing effect on the adaptive algorithm when implemented in practice.

In the single channel case, $P=M=L=1$, with $\alpha_{1}=\alpha_{2}$ and $\gamma_{1}=\gamma_{2}=1$, the algorithm reduces to the "RLMS" active control algorithm used by Eriksson [1], [2]. If there are no recursive coefficients, so that only FIR control filters are used, the algorithm also reduces to the Multiple Error LMS algorithm [12]. Equation (21) could thus be termed the Multiple Error RLMS algorithm.

A number of approximations have had to be made in the derivation above, some of which have been discussed in [21]-[24]. It is important to note, however, that exactly the same approximations are inherent in the single channel case [1], which has been found to perform well in a number of practical active control applications [2]. No additional approximations are required in the derivation of the multiple channel version of the algorithm, and our experience has been that provided a small leak is included, the algorithm is reasonably robust.

\section{EXPERIMENTAL RESULTS}

After a brief description of the experimental arrangement, we firstly present the results from experiments that should be directly comparable with the simulations carried out in Section II. Secondly, a more realistic case, where the primary noise is generated by a panel excited by a loudspeaker in an adjoining room, is investigated.

\section{A. Experimental Arrangement}

The heart of the arrangement was a TMS320c30 PC System Board and two 4 Channel Analog Interface Cards (from Loughborough Sound Images Ltd.) fitted into a PC, which was running the software controlling the $\mathrm{c} 30$ card. The two 4 Channel Cards, with a total of 8 inputs and 4 outputs, were used for the error sensors and secondary sources, respectively, whereas the reference signal was acquired with one of the BB PCM78 AD converters on the $c 30$ board. All inputs and outputs from the signal processing cards were low-pass filtered through a set of programmable filters with a cutoff frequency of $170 \mathrm{~Hz}$. The sources, both primary and secondary, were $4^{\prime \prime}$ loudspeakers in $2,2 l$ closed boxes, driven by power amplifiers, and the error sensors were $1 / 4$ in electret microphones. For the primary noise, the output from a pink noise generator was filtered through a high-pass and a low-pass filter section in order to obtain the desired shape of the primary noise spectrum.

Since the adaptive algorithm described above is rather complicated to implement in a computer program, it was decided to write the program for the $\mathrm{c} 30$ card in $\mathrm{C}$, and then handoptimize the generated assembler code. After some optimization it was possible to run a system with four secondary sources $(M=4)$, eight error sensors $(L=8)$, and with $I=J=K=32$ coefficients at a sampling frequency of $400 \mathrm{~Hz}$.

A typical experiment was carried out in three steps. First, the identification of the error paths was performed. All $M \cdot L=32$ error paths were modeled at the same time using 32 coefficient FIR filters, by means of four independent modeling noise generators feeding each secondary source, and the simple LMS adaptive identification algorithm. In prior comparisons of the 32-coefficient FIR estimate of an error path response with a 256-coefficient FIR estimate, it had been established that 32 coefficients was sufficient to model the error path to the accuracy required. Secondly, the control filters were adapted, using the previously identified error path models. Thirdly, the control filters were "frozen," by omitting the control filter update subroutine in the program, thus giving sufficient processing time for measurements of the residual signals to be taken by the PC.

The sum of the power spectral densities of each of the error signals was measured, while the controllers were "frozen," by Fourier transforming blocks of 512 contiguous samples of all 8 error signals, and adding together the square modulus of each spectrum. A specified number (generally 64 ) of added squared spectrums were averaged, finally converted to decibels, stored and denoted PSD8.

\section{B. Primary Source in the Receiving Room}

For the experiments reported on in this paragraph the window connecting the two rooms of the transmission loss measurement suite was filled with a box full of sand, and all sources and sensors were positioned in the receiving room as in the simulations presented above. Furthermore, it was ensured that the delay in the primary path was equal to the delay in the secondary paths so that the results from these experiments should be directly comparable with the results from the causality constrained theoretical optimization. Both FIR and IIR control filters were implemented in different experiments. The FIR control filters each had 32 coefficients and the IIR control filters each had 32 direct coefficients and 32 recursive coefficients.

The results in the "colored" noise case, after the control filters had been allowed to converge (which took about 20 seconds), are shown in Figs. 8 and 9. The first observation from these figures is that reductions are obtained which are comparable with the reductions predicted by the causality constrained optimization, cf. Fig. 7. The largest difference between the experimental and predicted reductions occurs in the control of the zeroth-order model below about $10 \mathrm{~Hz}$, which is negligibly excited experimentally and, therefore, does not significantly contribute to the mean square error. At higher frequencies the experimental system tends to reduce only the peaks in the spectra, at the cost of increasing the levels in the gaps between the peaks. However, this effect is in agreement with the simulations reported above and those reported by Joplin and Nelson [11].

At a second glance, the results in Figs. 8 and 9, obtained using the FIR and IIR control filters, may seem disappointingly alike. In order to stabilize the system with the IIR filters it 


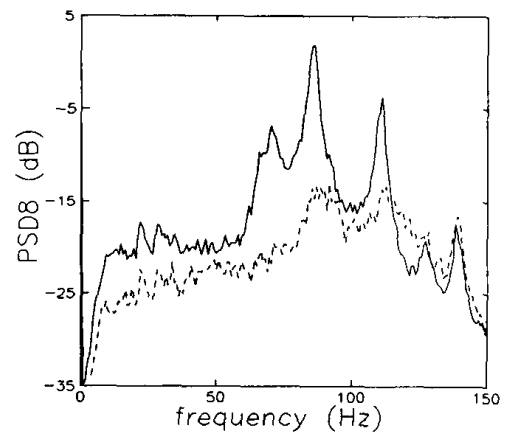

Fig. 8. Measurements of the sum of the power spectral densities of the 8 error signals (PSD8) before and - - - after control with FIR filters with 32 coefficients.

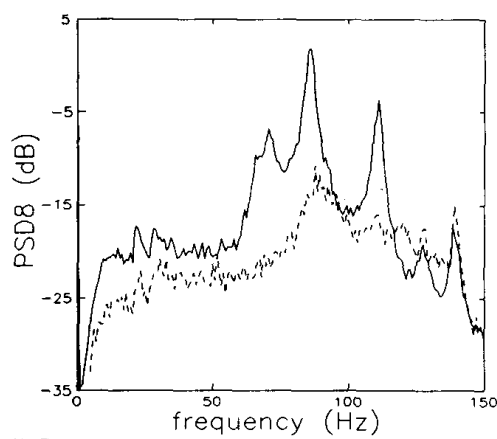

Fig. 9. Measurements of the sum of the power spectral densities of the 8 error signals (PSD8) before and - - - after control with IIR filters with 32 direct and 32 recursive coefficients.

was necessary to use a leak factor which was significantly less than unity $\left(\gamma_{2} \approx 0.98\right)$ in the recursive coefficient update equation. The recursive part of the filter thus never became very significant, as can be seen from the very modest improvement from Fig. 8 to Fig. 9, and from the magnitude of the recursive part of the converged filter coefficients, as shown in Fig. 10. The magnitude of the direct coefficients are of course dependent on all the gains in the control loop, whereas the recursive coefficients are relative to unity since they appear in the denominator of the controller transfer functions $H_{m}(z)$ in the following way:

$$
H_{m}(z)=\frac{a_{m 0}+a_{m 1} z^{-1}+\cdots+a_{m I-1} z^{-I+1}}{1-b_{m 1} z^{-1}-\cdots-b_{m K} z^{-K}} .
$$

The recursive coefficients seen from Fig. 10 are all quite small compared to unity and hence hardly contribute to the resulting controller transfer functions. Since a considerable restraint need to be placed on the recursive filter coefficients to ensure stability of the system, it seems as if the IIR filters are trying to converge to transfer functions with poles outside the unit circle. This is not entirely surprising, since the optimal unconstrained controllers are known to be noncausal. There are of course other, more sophisticated methods of stabilizing recursive filters than using a leak in the update equation, but unfortunately they are all rather expensive with respect to computation time, and hence they were not considered in this investigation.

It could be argued that the comparison between FIR filters with 32 coefficients and IIR filters with 32 direct and 32 recursive coefficients- 64 coefficients altogether-is unfair and that FIR filters with 64 coefficients should have been used instead, in order to have the same computational complexity in both filter types. However, the conclusion seems to be that FIR filters with 32 coefficients possess sufficient complexity to realize the optimal causal filters reasonably well, so that neither using 32/32-tap IIR filters nor 64-tap FIR filters would significantly improve the performance of the control system.

\section{Primary Source in the Adjoining Room}

In these experiments the primary noise field was generated by a loudspeaker in the adjoining source room and transmitted into the receiving room through a $2-\mathrm{mm}$ plywood panel inserted in the window between the two rooms. The dynamic characteristics of the panel now had a large influence on the sound field in the receiver room, and so the velocity distribution on the panel was measured [15]. These measurements suggested that at $20 \mathrm{~Hz}$ the panel had a resonance in which it moved as a piston in its mounting, whereas at $65 \mathrm{~Hz}$ the panel had a resonance which was similar to the first mode of a simply supported panel. An examination of the uncontrolled level measured at the microphones in the receiving room (solid curve in Fig. 11) reveals that the $20-\mathrm{Hz}$ piston mode of the panel is especially evident in the uncontrolled spectrum. This was expected to cause problems for several reasons. First, $20 \mathrm{~Hz}$ is between the natural frequencies of the $(0,0,0)$ and the $(0,0,1)$ acoustic modes of the receiving room, and from Fig. 1 it can be seen that only a limited reduction is possible at this frequency even in the unconstrained case. This is due to the fact that the sound field consists of contributions from many acoustic modes when excited at frequencies between resonances. Secondly, the algorithm was expected to expend a lot of "effort" on trying to control the large peak at $20 \mathrm{~Hz}$, without having any real chance of doing so, hence leaving the remaining field unaltered or perhaps even amplified. These expectations were confirmed by the initial experiments.

In order to be able to get better control of the $20-\mathrm{Hz}$ peak, one of the secondary sources was moved to the corner of the window, thus allowing it to couple into the room in a way similar to that of the primary source. The reductions at the control microphones for the "coloured" noise excitation using this secondary source arrangement are shown in Figs. 11 and 12, for the FIR and IIR controller cases, respectively. Several interesting observations can be made from these results. First, the reductions in both cases are not as large as those presented in the previous section, and in the areas between the peaks somewhat larger increases in noise levels are seen, unfortunately, probably due to the more complicated primary path. Secondly, it appears that the IIR filters perform far better than the FIR filters in this case. To verify that the IIR filters really were implementing impulse responses substantially longer than the 32 samples of the FIR filters, the impulse responses of two of the IIR control filters werre 


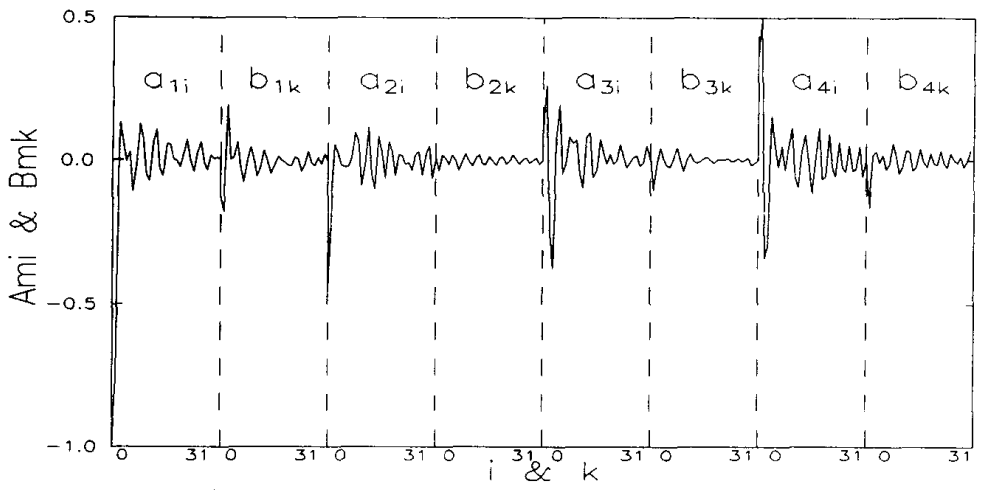

Fig. 10. The coefficients of the converged IIR control filters used for theexperiment that resulted in Fig. 9.

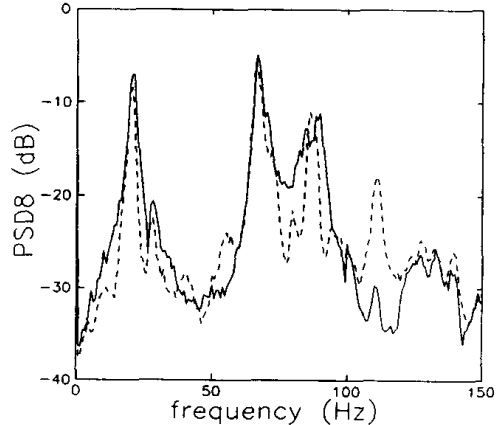

Fig. 11. Measurements of the sum of the power spectral densities of the 8 error signals (PSD8) before and - - - after control with FIR filters. The noise was generated by a panel excited from the adjoining room.

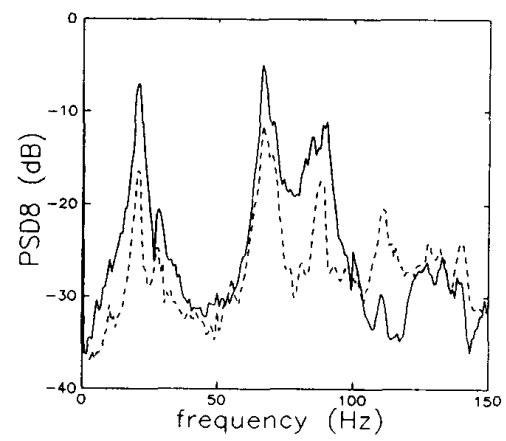

Fig. 12. Measurements of the sum of the power spectral densities of the 8 error signals (PSD8) before and - - - after control with IIR filters. The noise was generated by a panel excited from the adjoining room.

calculated and are shown in Figs. 13 and 14. Note that the two figures have different axes. Clearly the IIR filters are realizing filters with impulse responses significantly longer than 32 samples, especially for the secondary source in the window corner (corresponding to the impulse response shown in Fig. 14). This impulse response appears to be modeling the lightly damped impulse response of the panel, so that this secondary source can generate an acoustic output whose waveform best matches an inverted version of the waveform of the acoustic output of the panel.

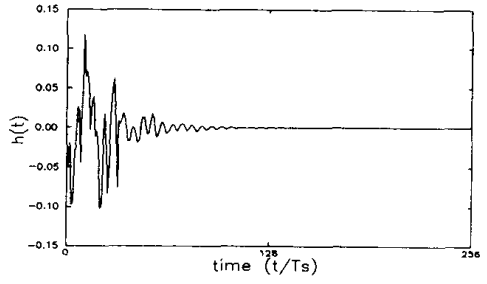

Fig. 13. Impulse response $h(t)$ of an IIR control filter feeding a secondary source on the floor, corresponding to the situation on Fig. 12. $T_{s}$ is the sampling period $(2.5 \mathrm{~ms})$.

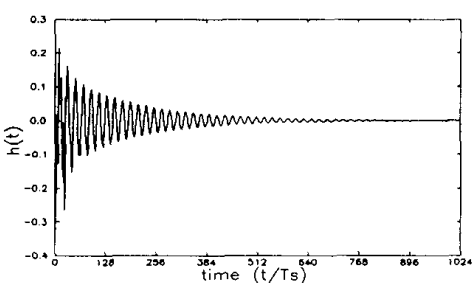

Fig. 14. Impulse response $h(t)$ of the IIR control filter feeding the secondary source positioned in the window close to the panel, corresponding to the situation on Fig. 12. $T_{s}$ is the sampling period $(2.5 \mathrm{~ms})$.

Again it can be argued that the 32/32-tap IIR filters should have been compared with 64-tap FIR filters, which undoubtedly would perform better than the 32-tap FIR filters, cf. Figs. 13 and 14. However, the exceptionally long response in Fig. 14 indicates that an IIR filter is still needed, at least for this secondary source.

\section{CONCLUSIONS}

In this paper an adaptive multichannel algorithm for IIR filters has been derived, and its application to active control of broadband random noise in a small reverberant room has been investigated experimentally.

The main conclusion is that adaptive multichannel active control using IIR filters is possible in practice. This has been demonstrated by the agreement between theoretical predictions of multichannel active control of broadband noise in a room and experimental results obtained by means of the derived algorithm. 
It appears, however, that when the noise in the controlled room is generated by a simple source, such as a loudspeaker, no substantial benefit is gained from using IIR filters instead of the more reliable FIR filters. However, if the noise is generated in a more complicated way which the controllers must mimic, such as when the noise is produced in an adjoining room and transmitted into the controlled room through a panel with a lightly damped dynamic behavior, then IIR filters have been found to be superior to FIR filters.

Furthermore, the derived algorithm has proved to be robust despite the stability problems inherent in the IIR filter structure. The stability has been achieved by introducing leak factors in the coefficient update equations.

It should finally be noted, however, that it is important to understand the physics of the problem for which multichannel active noise control is being considered, as was achieved in this case using the theoretical models described in section II. A great deal of time can be otherwise wasted in trying to solve a noise control problem using a method that is inherently incapable of providing the required noise reduction.

\section{ACKNOWLEDGMENT}

The authors would like to thank Chris Boucher and Trevor Sutton for assistance, and The Danish Research Academy for funding the first author's stay at the ISVR, during which this study was carried out.

\section{REFERENCES}

[1] L. J. Eriksson, M. C. Allie, and R. A. Greiner, "The selection and application of an IIR adaptive filter for use in active sound attenuation," IEEE Acoust., Speech, Signal Processing, vol. ASSP-35, pp. 433-437, 1987.

[2] L. J. Eriksson and M. C. Allie, "Use of random noise for on-line transducer modeling in an adaptive active attenuation system," J. Acoust. Soc. Amer., vol. 85, pp. 797-802, 1989.

[3] S. Laugesen, "Causality and stability problems in a simple active noise control system," in Proc. Inter.Noise 90, pp. 1281-1284, 1990.

[4] S. D. Goodman and S. S. Wise, "A discussion of commercial experience with active noise control on industrial fans and air handlers used for heating, ventilating and air conditioning," in Proc. Inter.Noise 90, pp. $797-800,1990$.

[5] S. J. Elliott, I. M. Stothers, P. A. Nelson, A. M. McDonald, D. C. Quinn, and T. Saunders, "The active control of engine noise inside cars," in Proc. Inter.Noise 88, pp. 987-990, 1988.

[6] S. J. Elliott, P. A. Nelson, I. M. Stothers, and C. C. Boucher," In-flight experiments on the active control of propeller-induced cabin noise," $J$. Sound Vib., vol. 140, pp. 219-238, 1990.

[7] L. J. Eriksson, M. C. Allie, R. H. Hoops, and J. V. Warner, "Higher order mode cancellation in ducts using active noise control," in Proc. Inter. Noise 89, pp. 495-500, 1989.

[8] A. M. McDonald, S. J. Elliott, and M. A. Stokes, "Active noise and vibration control within the automobile," in Proc. Int. Symp. on Active Control of Sound and Vibration, pp. 147-156, 1991.

[9] D. Guicking and $M$. Bronzel, "Multichannel broadband active noise control in small enclosures," in Proc. Inter.Noise 90, pp. 1255-1258, 1990.

[10] P. A. Nelson, J. K. Hammond, P. Joseph, and S. J. Elliott, "Active control of stationary random sound fields," J. Acoust. Soc. Amer., vol. 87 , no. 3, pp. $963-975,1990$.
[11] P. M. Joplin and P. A. Nelson, "Active control of low-frequency random sound in enclosures," J. Acoust. Soc. Amer., vol. 87, no. 6, pp. 2396-2404, 1990.

[12] S. J. Elliott, I. M. Stothers, and P. A. Nelson, "A multiple error LMS algorithm and its application to the active control of sound and vibration," IEEE Acoust., Speech, Signal Processing, vol. ASSP-35, pp. $1423-1434,1987$

[13] P. A. Nelson, A. R. D. Curtis, S. J. Elliott, and A. J. Bullmore, "The active minimization of harmonic enclosed sound fields, Part I: Theory," J. Sound Vib., vol. 117, no. 1, pp. 1-13, 1987.

[14] A. J. Bullmore, P. A. Nelson, A. R. D. Curtis, and S. J. Elliott, "The active minimization of harmonic enclosed sound fields, Part II: A computer simulation," J. Sound Vib., vol. 117, no. 1, pp. 15-33, 1987.

[15] S. Laugesen and S. J. Elliott, "A demonstration of multichannel broadband active noise control in a small reverberant room," ISVR, Memo. 709,1991

[16] S. P. Hough, "Some implications of causality in the active control of sound," Ph.D. dissertation, ISVR, Univ. of Southampton, Southampton, U.K., 1988.

[17] T. J. Sutton, S. J. Elliott, P. A. Nelson, and I. Moore, "The active control of road noise inside vehicles," Proc. Inter.Noise 90, pp. 1247-1250, 1990.

[18] S. J. Elliott and P. A. Nelson, "An adaptive algorithm for IIR filters used in multichannel active sound control systems," ISVR, Memo. 681, 1988.

[19] P. L. Feintuch, "An adaptive recursive LMS filter," Proc. IEEE, vol. 64, pp. $1622-1624,1976$.

[20] B. Widrow and S. D. Stearns, Adaptive Signal Processing. Englewood Cliffs, NJ: Prentice-Hall, 1985

[21] C. R. Johnson and M. G. Larimore, "Comments on and additions to "An adaptive recursive LMS filter," Proc. IEEE, vol. 65, pp. 1399-1402, 1977.

[22] B. Widrow and J. McCool, "Comments on "An adaptive recursive LMS filter", Proc. IEEE, vol. 65, pp. 1402-1404, 1977.

[23] J. J. Shynk, "Adaptive IIR filtering," IEEE ASSP Mag., pp. 4-21, Apr. 1989

[24] O. Macchi, "Common formalism for adaptive identification in signal processing and control," Proc. Inst. Elect. Eng., Part F, vol. 138, no. 4, pp. 295-307, 1991.

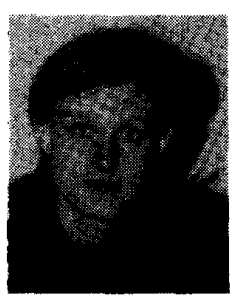

Søren Laugesen was born 1965 in Haderslev, Denmark. He received the M.Sc. degree in electrical engineering in 1989 and the Ph.D. degree on "Active Control of Acoustic Noise Using Adaptive Signal Processing" in 1992, both from the Acoustics Laboratory, Technical University of Denmark.

He is currently with Acoustics Laboratory, Technical University of Denmark on a project concerning the active control of tonal noise in a large chimney stack. His main interests are adaptive signal processing and active noise control, especially the modeling of the acoustical environment and the calculation of appropriate theoretical predictions of what can be achieved by an active control system.

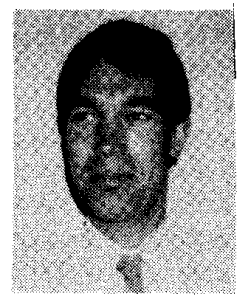

Stephen J. Elliott (M'83) was born in London, England, in 1954. He received the B.Sc. degree with joint honors in physics and electronics from the University of London, England, in 1976, and the Ph.D. degree from the University of Surrey, England, in 1979

After a period as a Research Fellow at the Institute of Sound and Vibration Research, University of Southhampton, England, and as a temporary Lecturer in the Physics Department of the University of Surrey, he was appointed as a Lecturer in ISVR under the SERC Special Replacement Scheme. His research interests include the physical and signal processing aspects of speech and active control. 